\title{
An Analysis of Energy Storage and Regulation
}

\author{
D. Fooladivanda, C. Rosenberg, and S. Garg \\ Dept. of Electrical and Computer Engineering, University of Waterloo, Canada \\ Email:\{dfooladi, cath, s6garg\}@uwaterloo.ca
}

\begin{abstract}
We focus on a region whose power system is controlled by an operator that relies on a regulation service to balance the total system supply to the total system load in quasi real-time. We consider the existing contractual framework in which a regulation unit declares its regulation parameters at the beginning of the contract, the operator guarantees that the regulation signals will be within the range of these parameters, and the regulation unit is rewarded proportionally to what it declares. Our purpose is twofold. We first want to obtain formulas for the regulation parameters that a unit with non-ideal storage should declare to the operator given its state of charge at the beginning of a contract. Second, we want to analytically quantify, ahead of time, the reward that such a unit could obtain in successive contracts by performing this regulation service. Since the state of charge at the beginning of a contract depends on what happened in the previous contract and, hence, is a random variable, we quantify this reward analytically using bounds and expectation. We then provide engineering insights by applying our results to three specific energy storage technologies that are often considered as candidates for regulation. In particular, we show the impact of the storage parameters and the length of one contract on the potential reward over a given period.
\end{abstract}

\section{NOMENCLATURE}

EST Energy storage technology

(S)RU (Storage) regulation unit

$e \quad$ Charging efficiency of the storage

$\eta \quad$ Depth of discharge of the storage

$B^{\prime}, B$ Capacity of the regulating storage, $B=\eta B^{\prime}(\mathrm{Wh})$

$\Delta_{c}, \Delta_{d}$ Max charging and discharging power limits (W)

$\alpha, \gamma \quad$ Charge/discharge time $\left(\alpha=\frac{B^{\prime}}{\Delta_{c}}, \gamma=\frac{B^{\prime}}{\Delta_{d}}\right)$ (hour)

$\beta \quad$ Discharge to charge ratio $\left(=\frac{\Delta_{d}}{\Delta_{c}}=\frac{\alpha}{\gamma}\right)$

$D, \delta \quad$ Contract and time-slot $(t s)$ duration

$\mathcal{K} \quad$ Set of $K$ time-slots $(D=K \delta)$

$R, r \quad$ Upward and downward regulation parameters (W)

$a_{n}, b_{n}$ Prices for each unit of upward and downward regulation per unit of time in contract $n\left(C_{n}\right)$

$f_{n}(\cdot) \quad$ Fixed part of regulation reward in $C_{n}$ (Dollar)

$F_{T} \quad$ Fixed part of regulation reward over a period of length $T$ hours (Dollar)

$s_{k} \quad$ Received regulation signal at $t s k(\mathrm{~W})$

$U_{n} \quad$ State of charge (SoC) at the beginning of $C_{n}(\mathrm{Wh})$

$b(k) \quad$ SoC of the storage at the end of $t s k(\mathrm{Wh})$

\section{INTRODUCTION}

Electrical grid operators have long performed what is known as "demand-following" which consists of scheduling generators with different characteristics (e.g., ramp rates) for supplying electricity to meet real-time demand. More recently, operators have started incorporating "supply-following" (also called "demand side management") techniques to take into account the introduction of large amounts of renewables into the grid. In either case, the grid needs to balance demand and supply at all times using services and providers that operate at different time-scales. Typically, the operator predicts the demand a day ahead and schedules some slow-ramping generators a day in advance. Quasi real-time adjustments are then made to maintain the balance between demand and supply and this is done using fast-ramping generators or flexible loads.

In this paper, we focus on the fastest time-scale, which typically ranges from tens of seconds to a few minutes. More specifically, we focus on a regulation service which balances the total system supply to the total system load in quasi realtime. This service is offered by regulating units (RU), typically generators (e.g., natural-gas-fired steam turbines, etc.), which can vary their supply rate in response to signals sent by the operator from every ten seconds to every few minutes. Because of the limited regulation capacity, ramping and ramp duration capabilities of each RU, it may be necessary for the operator to work concurrently with multiple RUs [1], using advanced algorithms to distribute the required regulation effort over all the RUs, while also considering their specific characteristics.

Several operators, including the California Independent System Operator (CAISO), have taken major steps to enable alternative technologies, such as energy storage technologies (EST), to be used for providing regulation services. ESTs that are typically considered for regulation services are flywheels, hydro-power plants, compressed air energy storage, and batteries [2]. Fast-response and energy recycling are two of the main characteristics of ESTs that are most often cited with reference to regulation [3]. While there are benefits to using storage technologies in regulation services, there are also specific challenges, such as their limited capacity, charging and discharging power limits, as well as inefficiency and selfdischarge. Our purpose is twofold. We first want to obtain formulas for the regulation parameters that a unit with nonideal storage should declare to the operator given its state of charge at the beginning of a contract. Second, we want to analytically quantify, ahead of time, the reward that such a unit could obtain in successive contracts by performing this regulation service.

To better present our contributions, we now describe the regulation service along with the existing contractual framework assuming a generic regulation unit ${ }^{1}$. Every $D$ units of time, a new regulation contract starts. This contract has been negotiated a few units of time ahead. As a result of this negotiation, the operator and the RU agree on the regulation parameters $r$ and $R(r, R \in \mathbb{R}, r<R)$. The RU commits to provide any power in the range $[r, R]$ in response to regulation signals sent by the operator ( $R$ and $r$ are in Watts). During the contract, the operator will send regulation signals $s_{k}$ to the

\footnotetext{
${ }^{1}$ We use the term "regulation unit (RU)" to refer to a generic unit and "storage regulation unit (SRU)" to refer to a unit using a non-ideal storage.
} 
RU in time-slot $k \in\{1, \ldots, K\}$ with $D=K \delta$ (where $\delta$ is the duration of a time-slot) ensuring that:

$$
r \leq s_{k} \leq R
$$

The RU must supply constant power $s_{k}$ during time-slot $k$ if $s_{k}$ is positive, and draw constant power $\left(-s_{k}\right)$ from the grid if it is negative. Note that in the existing contractual framework, the operator can generate any sequence of regulation signals, as long as the inequality above is obeyed. The RU is rewarded for its flexibility in terms of $R$ and $r$, and for what it actually supplies/draws during the contract. There is a penalty to pay if the RU cannot supply/draw as agreed. The second part of the reward is not known at the beginning of a contract, so typically, an RU will select its parameters $r$ and $R$ so as to maximize the fixed part of the reward, which we call $f(R, r)$, while keeping the risk of a regulation failure very small or close to zero ${ }^{2}$. In the following, when we refer to reward, we only refer to the fixed part of the reward. In addition, we assume that the RU declares some parameters $r$ and $R$ at the beginning of a contract and that the operator accepts these parameters and hence pays $f(R, r)$ to the RU.

Under this contractual framework, a regulation unit with non-ideal storage (SRU) would declare its regulation parameters $R$ and $r$ as a function of the size of its storage, of its charging and discharging power limits and of its efficiency/selfdischarge, as well as, more critically, as a function of its state of charge (SoC) at the beginning of the contract. Providing formulas to help the SRU declare its regulation parameters as a function of the SoC at the beginning of a contract is our first purpose. Our second purpose is to help the SRU determine, ahead of time, the storage technology, sizing, and contract duration that would yield the maximum reward (in terms of the best and worst-case rewards as well as the average reward) over longer periods. The SRU does not have direct control of the contract duration but might try to influence (i.e., negotiate with) operators and policy makers if the current contract duration is too unfavorable. Dealing with longer periods, i.e., over multiple contracts, is challenging because the SoC at the end a contract is a random variable (since it depends on the regulation signals sent during the contract), and consequently, the initial SoC in the next contract is also a random variable. Our contributions are:

1) We first focus on a single contract and formulate the reward maximization problem to compute the regulation parameters $R$ and $r$, when considering a non-ideal SRU, assuming that the initial SoC is known and that we want to keep the risk of a regulation failure equal to zero. We derive simple closed formulas.

2) Then, we focus on a sequence of $N$ contracts at the beginning of the first contract and compute analytical upper and lower bounds on the reward that could be obtained out of these $N$ contracts. We also propose an approximate formula for the expected reward that can be obtained from $N$ contracts.

\footnotetext{
${ }^{2}$ We choose the values of the regulations parameters such that the RU can always supply and draw, i.e., the RU does not take any risks, and hence does not pay any penalty.
}

3) We obtain numerical results for three specific ESTs that are often considered as candidates for regulation. We first validate our approximation for the expected reward over $N$ contracts. We then obtain engineering insights by comparing the rewards that these three technologies can bring for different values of some critical parameters.

The rest of the paper is organized as follows: Section II presents the literature. The system model is introduced in Section III. Section IV presents our analytical results for a single contract while Section $\mathrm{V}$ present the results for $N$ successive contracts. Numerical results are presented in Section VI.

\section{LITERATURE BACKGROUND}

In conventional regulation services, grid frequency is maintained by generators and loads that respond to the regulation signals sent by the operator. A comprehensive overview of conventional frequency regulation services is provided in [4].

Practical experiments have shown that ESTs can provide fast and accurate frequency regulation services [6], [5]. Operational benefits and costs of using ESTs for frequency regulation are quantified, via simulation, in [1], [2], [6]. However, challenges remain that prevent the use of ESTs for the regulation market, e.g., payment policy [7] and energy storage management [3], [8].

As explained earlier, SRUs are paid for their flexibility in terms of their charging/discharging power limits, and what they actually supply/draw during a contract [7]. To maximize the fixed part of the reward, typically, SRUs commit to providing/absorbing energy with their rated power output [1], [8]. When an energy storage unit provides regulation services without any control over its maximum charging/discharging power limits, after a short time, it may deplete its energy or be fully charged, which results in regulation failures and revenue losses. In [8], Lu et al. show, via simulation, that the risk of a regulation failure is not zero when a flywheel provides regulation services by itself without any control over its charging/discharging power limits.

Over-dimensioning storage resources is another way to decrease the risk of a regulation failure in a contracted regulation service. In [9], the authors propose a method for dimensioning a storage resource to provide frequency regulation. The proposed method uses historical frequency measurements to compute the minimum possible capacity.

Extensive work has been done to study how storage could provide frequency regulation but mostly via simulation. Previous papers do not compare the performance of different ESTs in providing regulation services in consecutive contracts. To our knowledge, our work is the first that computes the optimal values of an SRU's flexibility (i.e., $R$ and $r$ ) in terms of its charging/discharging power limits, so that it can respond to all possible sequences of regulation signals without any failure such that the fixed part of its reward is maximized and the first to propose analytical formulas for the expected reward over consecutive contracts.

\section{System Model}

We consider an SRU that has a non-ideal storage of size $B^{\prime}$ (Watt-hour), with charging efficiency $0<e \leq 1$, maximum charging and discharging power limits $\Delta_{c}$ and $\Delta_{d}$ (Watt), 
respectively, and a depth of discharge (DoD) $\eta$ (i.e., the available capacity is $\left.B=\eta B^{\prime}\right)$. We assume that the storage power leakage (i.e., self-discharge) is negligible, and that the discharging efficiency is equal to one. Typically, discharging power limits are greater than the charging power limits [10], [11] and hence, in the following, we assume that $\Delta_{d} \geq \Delta_{c}$.

Different storage technologies can be characterized by the range of values taken by the following parameters: charging efficiency, charge time, and discharge to charge ratio [10], [11]. The charge time denoted by $\alpha$ is defined as the ratio of the storage size (i.e., $B^{\prime}$ ) to its maximum charging power limit $\left(\Delta_{c}\right)$, while the discharge time $\gamma$ is the ratio of the storage size (i.e., $B^{\prime}$ ) to its maximum discharging power limit. We also define the discharge to charge ratio $\beta$ as the ratio of the discharging power limit of the storage to its charging power limit. Note that $\gamma=\frac{\alpha}{\beta}$.

Let us assume that the time is slotted in time-slot ( $t s)$ of size $\delta$, and that the duration of each contract is $K$ time-slots (i.e., $D=K \delta$ ). We assume that the fixed part of the reward in terms of $R_{n}$ and $r_{n}$ in each contract $C_{n}$ is a linear function of $R_{n}$ and $r_{n}$, i.e., $f\left(R_{n},\left|r_{n}\right|\right)=\left(a_{n} R_{n}+b_{n}\left|r_{n}\right|\right) D$ in dollars where $a_{n} \geq 0$ and $b_{n} \geq 0$ denote the prices for each Watt of upward and downward regulation per unit of time, respectively.

Let us focus on one contract (we omit the index $n$ ). The SRU commits to provide any power in the range $[r, R](r \leq 0$ and $R \geq 0$ ), in response to the regulation signal $s_{k}$, that the operator will send to the SRU at time-slot $k(1 \leq k \leq K)$. The SRU must supply constant power $s_{k}$ during time-slot $k$ if $s_{k}$ is positive and must draw constant power $\left(-s_{k}\right)$ from the grid if it is negative. During the contract, the SRU will receive a sequence of regulation signals $\left\{s_{1}, \cdots, s_{K}\right\}$. Let $b(k)$ denote the SoC of the storage at the end of $t s k$, it evolves as follows:

$$
b(k)=b(k-1)-\delta\left[s_{k}\right]^{+}+e \delta\left[-s_{k}\right]^{+} \quad \forall k \in \mathcal{K}
$$

where $b(0)=U, U$ is the initial SoC, $\mathcal{K}=\{1, \ldots, K\}$, and $[x]^{+}$is equal to $x$ if $x \geq 0$; otherwise, it is zero.

The SRU needs to make sure when choosing $r$ and $R$ that the following constraints are satisfied irrespective of the regulation signals being produced.

$$
\begin{aligned}
& 0 \leq b(k) \leq B \quad \forall k \in \mathcal{K} \\
& {\left[-s_{k}\right]^{+} \leq \Delta_{c} \quad \forall k \in \mathcal{K}} \\
& {\left[s_{k}\right]^{+} \leq \Delta_{d} \quad \forall k \in \mathcal{K} .}
\end{aligned}
$$

Note that the only constraint imposed on the regulation signals for the existing framework is $r \leq s_{k} \leq R$ for all $k$. This translates directly into: $R \leq \Delta_{d}$ and $|r| \leq \Delta_{c}$.

In the following section, we first focus on a single contract and then on multiple consecutive contracts.

\section{Analytical Results: Single Contract}

Consider a single contract and assume that the SRU knows its SoC at the beginning of the contract (we call it $U$ ). The SRU has to choose its parameters $R$ and $r$ so that i) it can respond to all possible sequences of regulation signals $\left\{s_{k}\right\}$ without any failure, ii) its reward $f(R,|r|)$ is maximized, and iii) the SoC at the end of the contract is between 0 and $B$.
Given a pair $(R, r)$ with $0 \leq R \leq \Delta_{d}$ and $0 \leq(-r) \leq \Delta_{c}$, define the polyhedron $F(R, r)$ as follows ${ }^{3}$ :

$$
F(R, r)=\left\{\pi \in \mathbb{R}^{K} \mid \pi=\left[s_{1}, \ldots, s_{K}\right]^{t}, r \leq s_{k} \leq R\right\} .
$$

The pair $(R, r)$ is feasible (i.e., it can be selected by the SRU without any risk of a regulation failure) if (2) is satisfied for all $\pi \in F(R, r)$. Among all feasible pairs $(R, r)$, we are interested in finding the one that maximizes $f(R,|r|)=$ $(a R+b|r|) D$. Let us consider the two following sequences of regulation signals:

$$
\pi_{1}: s_{k}=r \quad \forall k \in \mathcal{K} ; \pi_{2}: s_{k}=R \quad \forall k \in \mathcal{K}
$$

Proposition 1 establishes that pair $(R, r)$ is feasible if and only if the value of $b(K)$ obtained by the sequence $\pi_{1}$ (resp. $\pi_{2}$ ) is less than or equal to $B$ (resp. greater than or equal to 0 ). We present the following result without proof due to lack of space.

Proposition 1. Given $\delta, K$, e, and B, pair $(R, r)$ is feasible for the existing contractual framework if and only if each of the sequences $\pi_{1}$ and $\pi_{2}$ yield $0 \leq b(K) \leq B$.

Clearly sequences $\pi_{1}$ and $\pi_{2}$ can be seen as worst-case sequences. Constraint (2) is satisfied for sequence $\pi_{1}$ iff

$$
0 \leq|r| \leq \frac{B-U}{e D} \text {. }
$$

Similarly, constraint (2) is satisfied for sequence $\pi_{2}$ iff

$$
\frac{U-B}{D} \leq R \leq \frac{U}{D}
$$

Hence, (2) is satisfied for all $\pi \in F(R, r)$ (i.e., $(R, r)$ is feasible) if and only if (6) and (7) are satisfied. Recall that $0 \leq R \leq \Delta_{d}$ and $0 \leq(-r) \leq \Delta_{c}$. Therefore, given $B, U, D$, $\Delta_{d}, \Delta_{c}$, and $e$, pair $(R, r)$ is feasible if and only if:

$$
\begin{aligned}
0 \leq R \leq R^{(1)} & \triangleq \min \left(\Delta_{d}, \frac{U}{D}\right) \\
|r| & \leq r^{(1)} \triangleq \min \left(\Delta_{c}, \frac{B-U}{D e}\right) .
\end{aligned}
$$

Clearly, $r^{(1)}$ and $R^{(1)}$ are the values of $r$ and $R$ that maximize $f(R,|r|)$ as long as $f$ is increasing in its arguments. Note that $r^{(1)}$ and $R^{(1)}$ are functions of $U$, the SoC at the beginning of the contract. Hence, computing beforehand the reward in a multiple contract setting is not straightforward since estimating the $\mathrm{SoC}$ at the end of a contract depends on the regulation signals that will be sent.

\section{Analytical Results: Multiple Contracts}

Let us assume that the SRU bids for a set of $N$ consecutive contracts for a total duration of $T=N D$ time units, and that $U_{n}$ denotes the SoC at the beginning of contract $n$, i.e., the SoC at the end of $C_{n-1}$. We assume that $U_{1}$ is fixed and known a priori. We want to characterize a priori the reward $F_{T}=\sum_{n=1}^{N} f_{n}\left(R_{n},\left|r_{n}\right|\right)$ that the SRU can expect over the $N$ contracts. The index $n$ in $f_{n}(\cdot)$ reflects the fact that the prices for each unit of upward regulation $\left(a_{n}\right)$ and downward

\footnotetext{
${ }^{3}$ The superscript " $t$ " denotes the transpose operation.
} 
regulation $\left(b_{n}\right)$ might be different for each contract. Note that the reward $F_{T}$ is a random variable whose value depends on the sequences of regulation signals being produced by the operator. These sequences are unknown a priori and hence we do not know the SoC at the beginning of $C_{n}$ for $n>1$. We first characterize the reward by providing upper and lower bounds and we then compute an approximation of its average. A. Bounds

To obtain upper and lower bounds on the reward $F_{T}=$ $\sum_{n=1}^{N} f_{n}\left(R_{n}, r_{n}\right)$ that the SRU can obtain over the $N$ contracts, we first consider a single contract $n$ and assume that we do not know the SoC $U_{n}$ at the beginning of that contract. However, we know that $U_{n} \in[0, B]$. Proposition 2 derives an upper bound $\overline{f_{n}}$ and a lower bound $f_{n}$ for the reward for that single contract which are independent of the value of the initial SoC, i.e., $U_{n}$.

Typically, the parameters $\alpha, \beta, e$ and $\eta$, are quasi-constant for a given technology, and hence $\Delta_{c}$ and $\Delta_{d}$ are quasilinearly proportional to the storage capacity. In the following, we assume that these parameters are constant. To understand the impact of the available capacity $B$ and the contract duration $D$ on the lower and upper bounds of the reward, we present our analytical results in terms of the storage parameters $\alpha, \beta, B, e$, and $\eta$. The proof of Proposition 2 is provided in the appendix.

Proposition 2. Let $a_{n} \geq 0$ and $b_{n} \geq 0$ denote the prices for each unit of upward and downward regulation in contract $n$. Given $\alpha, \beta, e, D, B$, and $\eta$, the SRU's reward $f_{n}\left(R_{n}, r_{n}\right)$ is bounded as follows:

$$
\underline{f_{n}} \leq f_{n}\left(R_{n}, r_{n}\right) \leq \overline{f_{n}}
$$

where:

1) If $D \geq \eta \frac{\alpha}{e}$, then

$$
\underline{f_{n}}=B \min \left\{a_{n}, \frac{b_{n}}{e}\right\}, \overline{f_{n}}=B \max \left\{a_{n}, \frac{b_{n}}{e}\right\} .
$$

2) If $\gamma \eta \leq D<\eta \frac{\alpha}{e}$, then

$$
\underline{f_{n}}=B D \min \left\{\frac{a_{n}}{D}, \frac{b_{n}}{\alpha \eta}\right\}, \overline{f_{n}}=B D \max \left\{\frac{W_{n}}{\alpha \eta}, \frac{a_{n}}{D}\right\} .
$$

3) If $\eta \frac{\alpha}{e+\beta} \leq D<\gamma \eta$, then

$$
\underline{f_{n}}=D \frac{B}{\alpha \eta} \min \left\{a_{n} \beta, b_{n}\right\}, \overline{f_{n}}=D \frac{B}{\alpha \eta} \max \left\{Q_{n}, W_{n}\right\} .
$$

4) If $D<\eta \frac{\alpha}{e+\beta}$, then

$$
\underline{f_{n}}=D \frac{B}{\alpha \eta} \min \left\{a_{n} \beta, b_{n}\right\}, \overline{f_{n}}=D \frac{B}{\alpha \eta}\left(a_{n} \beta+b_{n}\right) .
$$

where $Q_{n}=\beta\left(a_{n}+\frac{b_{n}}{e}\left(\frac{\gamma \eta}{D}-1\right)\right)$ and $W_{n}=$ $\left(b_{n}+a_{n}\left(\frac{\eta \alpha}{D}-e\right)\right)$.

Given $\alpha, \beta, e, D$, and $B$, the reward $F_{T}$ over the $N$ contracts (recall that $T=N D$ ) is bounded as follows:

$$
\sum_{n=1}^{N} \underline{f_{n}} \leq F_{T} \leq \sum_{n=1}^{N} \overline{f_{n}}
$$

Table I gives the lower and upper bounds over $N$ contracts given that $a_{n}=a$ and $b_{n}=b$.
Engineering insights based on the bounds: Our analytical results in Table I show that the upper and lower bounds are proportional to $B$ while their relationships with $D$ are more complex. To understand the impact of $D$, let us consider the following three storage technologies, namely two battery technologies (Lithium-ion and Lead-acid) and a flywheel technology ${ }^{4}$. The ranges of values for $\alpha, \beta, \eta$, and $e$ for these technologies are shown in Table II. Typically, for a given technology, the parameters $\alpha$ and $\beta$ are quasi constant (i.e., they do not change when we change the storage capacity). Hence, the only free parameter from the standpoint of the SRU is the storage capacity $B=\eta B^{\prime}$ but a SRU could influence the operator to negotiate a contract duration $D$ which is favorable to its storage technology. Using Tables I and II, we can state the following (assuming $a_{n}=a, b_{n}=b$ and $T$ constant):

- Flywheel: for this technology, $\eta \frac{\alpha}{e}$ is of the order of a few minutes (less than five) and since $D$ the contract duration will most probably be larger than five minutes we are in Case 1 and so the SRU's minimum reward during the period of $T$ hours is inversely proportional to $D$ when $T$ is constant. Hence, an SRU using a flywheel would benefit a lot (in terms of reward) from a shorter contract duration (e.g., 10 minutes).

- Lithium-ion: Depending on the value of $D$, we will be in Case 2 or Case 3 and hence it is not always clear that the SRU would benefit from a smaller contract duration. For example, if we are in Case 2, a shorter contract duration will only help as long as $\frac{a}{D}<\frac{b}{\eta \alpha}$.

- Lead-acid: Depending on the value of $D$, we will be in Case 3 or Case 4. For example, if $D<40$ minutes, then we are in Case 4 and the minimum and maximum rewards are independent of $D$.

\section{B. Average Reward}

Another way to characterize the potential reward from the $N$ contracts is to compute its expected value $E\left\{F_{T}\right\}$. Let $E_{n}$ denote the expected value of the reward in contract $n$ (i.e., $\left.E_{n}=E\left\{f_{n}\left(R_{n},\left|r_{n}\right|\right)\right\}\right)$. Clearly, $R_{n}$ and $r_{n}$ are a function of $U_{n}$, and hence are random variables whose potential values depend on the past history. The expected reward over the $N$ contracts is $E\left\{F_{T}\right\}=E_{1}+\sum_{n=2}^{N} E_{n}$ (the reason we differentiate between $E_{1}$ and $E_{n}, n \geq 2$ is that we know that $U_{1}$ while we do not know $U_{n}$ for $n \geq 2$ ). Recall that

$$
E_{1}=D\left(a_{1} \min \left\{\Delta_{d}, \frac{U_{1}}{D}\right\}+b_{1} \min \left\{\Delta_{c}, \frac{B-U_{1}}{D e}\right\}\right) .
$$

Let $g_{n}(\cdot)$ denote the probability distribution function of $U_{n}$ over the range $[0, B]$. We can compute $E_{n}$ as follows:

$$
E_{n}=\int_{0}^{B} f_{n}\left(R_{n}(x),\left|r_{n}(x)\right|\right) g_{n}(x) d x, \quad n \geq 2 .
$$

where the notation $R_{n}(x)$ (resp. $r_{n}(x)$ ) means $R_{n}$ (resp. $r_{n}$ ) given $U_{n}=x$. Note that given $U_{n}=x$, we can use (8) and (9) to compute $R_{n}(x)$ and $r_{n}(x)$.

Determining the distribution of $U_{n}$ over $[0, B]$ is challenging and is highly dependent on the distribution of the regulation signals in contract $C_{n-1}$ over the range $\left[r_{(n-1)}, R_{(n-1)}\right]$.

\footnotetext{
${ }^{4}$ Note that self-discharge is probably not negligible for Flywheels.
} 
TABLE I

LOWER AND UPPER BOUNDS ON THE REWARD $F_{T}$ WHEN $a_{n}=a, b_{n}=b$, AND $T=N D$.

\begin{tabular}{|l|c|c|}
\hline Conditions & Lower bound on $F_{T}$ & Upper bound on $F_{T}$ \\
\hline Case 1: $D \geq \eta \frac{\alpha}{e}$ & $\frac{T B}{D} \min \left\{a, \frac{b}{e}\right\}$ & $\frac{T B}{D} \max \left\{a, \frac{b}{e}\right\}$ \\
\hline Case 2: $\gamma \eta \leq D<\eta \frac{\alpha}{e}$ & $T B \min \left\{\frac{a}{D}, \frac{b}{\eta \alpha}\right\}$ & $T B \max \left\{\frac{W}{\eta \alpha}, \frac{a}{D}\right\}$ \\
\hline Case 3: $\eta \frac{\alpha}{(e+\beta)} \leq D<\gamma \eta$ & $\frac{T B}{\eta \alpha} \min \{a \beta, b\}$ & $\frac{T B}{\eta \alpha} \max \{Q, W\}$ \\
\hline Case 4: $D<\eta \frac{\alpha}{(e+\beta)}$ & $\frac{T B}{\eta \alpha} \min \{a \beta, b\}$ & $\frac{T B}{\eta \alpha}(a \beta+b)$ \\
\hline \hline \multicolumn{2}{|c|}{$Q=\beta\left(a+\frac{b}{e}\left(\frac{\gamma \eta}{D}-1\right)\right), W=\left(b+a\left(\frac{\alpha \eta}{D}-e\right)\right)$} \\
\hline
\end{tabular}

TABLE II

STORAGE CHARACTERISTICS [10]- [11]

\begin{tabular}{|c|c|c|c|}
\hline Storage technology & Lead-acid & Lithium-ion & Flywheel \\
\hline \hline Charging efficiency e & 0.75 & 0.85 & 0.95 \\
\hline Charge time $\alpha$ & $8-16 \mathrm{~h}$ & $2-4 \mathrm{~h}$ & $1-3 \mathrm{~min}$ \\
\hline Discharge time $\gamma$ & $48-96 \mathrm{~min}$ & $24-48 \mathrm{~min}$ & $1-3 \mathrm{~min}$ \\
\hline $\begin{array}{c}\text { Discharge power limit } \\
\text { to charge power limit ratio }\end{array}$ & 10 & 5 & 1 \\
\hline DoD $\eta$ & 0.8 & 0.8 & 1 \\
\hline
\end{tabular}

If we assume that the regulation signals in contract $n$ are uniformly distributed in $\left[r_{n}, R_{n}\right]$, we can show that $U_{n}$ is distributed according to an Irwin-Hall distribution when the SRU is using an ideal battery, but it is hard to analytically determine the probability distribution function of $U_{n}$ for a non-ideal battery (i.e., when $e<1$ ). Hence, we propose a method to approximate the average reward $E\left\{F_{T}\right\}$ over the $N$ contracts. In the following, $\widetilde{X}$ represents an approximation of the real-valued variable $X$. In a first step, we begin by assuming that:

$$
\frac{\widetilde{E_{n}}}{D}=a_{n} \min \left\{\Delta_{d}, \frac{E\left\{U_{n}\right\}}{D}\right\}+b_{n} \min \left\{\Delta_{c}, \frac{B-E\left\{U_{n}\right\}}{D e}\right\}_{(10)}
$$

In the second step, we compute an estimate for $E\left\{U_{n}\right\}$. We have tried multiple approximations. The first one was to assume that $U_{n}$ is uniformly distributed between 0 and $B$. This worked very well for two of the three storage technologies that we studied, but not for the third one. The one that provides the most accurate results for all three technologies involves approximates $E\left\{U_{n}\right\}$ as $\widetilde{E\left\{U_{n}\right\}}=\frac{X_{n}+Y_{n}}{2}$, where $X_{n}$ and $Y_{n}$ are given in Table III. Here $X_{n}$ and $Y_{n}$ are the minimum and maximum SoCs at the end of contract $n$, averaged over all initial SoCs at the beginning of contract $n$. The distribution of the initial $\mathrm{SoC}$ is approximated as a uniform distribution between its minimum and maximum values.

Using this approximate method, we can iteratively compute $\widetilde{E\left\{U_{n}\right\}}$ for all $n$. After computing $\widetilde{E\left\{U_{n}\right\}}$ for all $n$, we can easily compute $\widetilde{E_{n}}$ using (10). Finally, we compute our approximation of $E\left\{F_{T}\right\}$ which is equal to $E_{1}+\sum_{n=2}^{N} \widetilde{E_{n}}$. In the next subsection, we validate our approximate method, and provide some engineering insights using numerical results.

\section{Numerical Results: VALIDATION AND ENGINEERING INSIGHTS}

We consider the three storage technologies discussed earlier, i.e., Lithium-ion, Lead-acid, and Flywheel. Focusing on a period of ten hours (i.e., $T=10 h$ ), we take $K=60$, $U_{1}=\frac{B}{2}, a_{n}=b_{n}=1(\$ /(\mathrm{h} \mathrm{MW}))$, and $\alpha$ to be equal

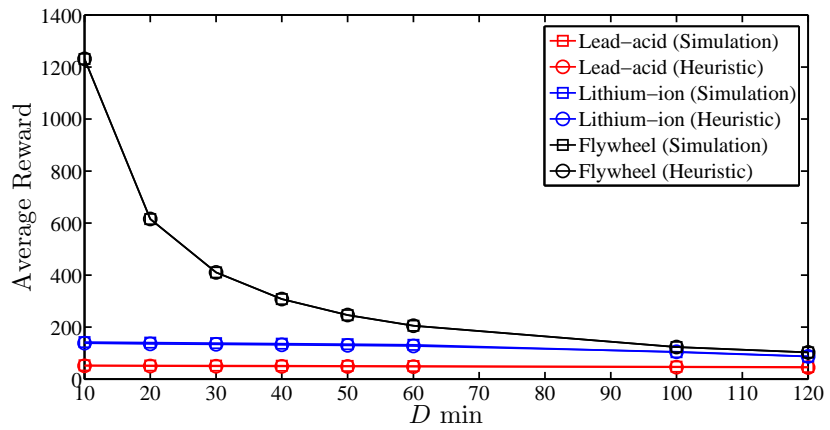

Fig. 1. The expected value of the reward $E\left\{F_{T}\right\}$ (in $\$$ ) as well as our approximation of $E\left\{F_{T}\right\}$ as a function of $D$ for $B=20 M W h$ when $\alpha=10 h$ (Lead-acid), $\alpha=3 h$ (Lithium-ion), $\alpha=2 \min$ (Flywheel), $T=10 h, K=60$, and $a_{n}=b_{n}=1 \$ /(\mathrm{h} \mathrm{MW})$.

to 10 hours, 3 hours, and 2 minutes for Lead-acid, Lithiumion, and Flywheel, respectively. To validate our approximation on the expected reward over $T$, we simulate regulation signals in contract $C_{n}$ that are uniformly distributed in the range $\left[r_{n}\left(U_{n}\right), R_{n}\left(U_{n}\right)\right]^{5}$. More precisely, given $U_{1}=\frac{B}{2}$, we compute $R_{1}$ and $r_{1}$, and generate $K$ (uniformly distributed in $\left.\left[r_{1}, R_{1}\right]\right)$ regulation signals for $C_{1}$. Given $U_{1}$ and the regulation signals in $C_{1}$, we can compute $U_{2}$, and then $r_{2}\left(U_{2}\right)$, and $R_{2}\left(U_{2}\right)$, and hence we can compute the reward in $C_{2}$. By doing the same process for $C_{3}, \cdots, C_{n}$, we can compute the reward over the $N$ contracts for one realization of regulation signals. We compute the average reward over 3000 realizations given $D$ and $B$. An alternate approach would have been to obtain the regulation signals $\left\{s_{k}\right\}$ from a dataset, but these would correspond to specific values of parameters $r_{n}$ and $R_{n}$, different from the optimized values that we derive in this paper. Thus we would have been limited in our ability to study the impact of different battery technologies, contract durations and battery capacities on the average SRU reward.

To validate our approximate method and to understand the impact of $D$ on the average reward, we compute our approximation of the average reward as well as the average reward over 3000 realizations for different values of the contract duration $D$ and $B=20 M W h$. Our numerical results in Fig. 1 show that:

- Our approximation method works very well.

- $E\left\{F_{T}\right\}$ is non-increasing in $D$ for the 3 technologies.

- For Lead-acid and Lithium-ion, the expected value is not significantly impacted by the value of $D$.

- With Flywheel, the contract duration $D$ has a significant impact on the SRU's average reward. For $D=10 \mathrm{~min}$, we saw a gain of $500 \%$ (with respect to $D=1 h$ ).

- Flywheel can achieve a higher reward for the same value of $D$ than Lead-acid and Lithium-ion can. The difference in $E\left\{F_{T}\right\}$ is minor for $D \geq 50 \mathrm{~min}$ and significant for $D \leq 30 \mathrm{~min}$.

We obtained the same results for different values of $B$.

To understand the impact of the storage capacity $B$ on the SRU's reward $E\left\{F_{T}\right\}$, we computed the average reward over 3000 realizations (not represented in the figure because they are very indistinguishable from the approximations), our

\footnotetext{
${ }^{5}$ Clearly, different signal distributions would yield different results
} 
TABLE III

The HeUristic PARAMETERS $X_{(n+1)}$ AND $Y_{(n+1)}$ FOR CONTRACT $(n+1)$

\begin{tabular}{|c|c|c|}
\hline Conditions & $X_{(n+1)}$ & $Y_{(n+1)}$ \\
\hline Case 1: $D \geq \eta \frac{\alpha}{e}$ & 0 & $B$ \\
\hline Case 2: $\gamma \eta \leq D<\eta \frac{\alpha}{e}$ & ${ }^{2}$ & $\begin{array}{cl}E\left\{U_{n}\right\}+e D \Delta_{c}, & \text { if } Y_{n} \leq Z_{c} \\
B-\frac{Z_{c}{ }^{2}}{2 Y_{n}}, & \text { if } Y_{n} \geq Z_{c}\end{array}$ \\
\hline Case 3: $\eta \frac{\alpha}{(e+\beta)} \leq D<\gamma \eta$ & $\begin{array}{cc}0, & \text { if } \quad Y_{n} \leq Z_{d} \\
H_{n}, & \text { if } \quad X_{n} \leq Z_{d} \\
E\left\{U_{n}\right\}-D \Delta_{d}, & \text { if } \quad X_{n} \geq Z_{d} \\
\end{array}$ & $\begin{array}{cl}E\left\{U_{n}\right\}+e D \Delta_{c}, & \text { if } Y_{n} \leq Z_{c} \\
G_{n}, & \text { if } \quad X_{n} \leq Z_{d} \leq Y_{n} \\
B, & \text { if } X_{n} \geq Z_{c}\end{array}$ \\
\hline Case 4: $D<\eta \frac{\alpha}{(e+\beta)}$ & $\begin{array}{cl}0, & \text { if } \overline{Y_{n} \leq Z_{d}} \\
H_{n}, & \text { if } \quad X_{n} \leq \bar{Z}_{d} \leq Y_{n} \\
E\left\{U_{n}\right\}-D \Delta_{d}, & \text { if } \quad X_{n} \geq \bar{Z}_{d} \\
\end{array}$ & $\begin{array}{cl}E\left\{U_{n}\right\}+e D \Delta_{c}, & \text { if } Y_{n} \leq Z_{c} \\
G_{n}, & \text { if } X_{n} \leq \bar{Z}_{c} \leq Y_{n} \\
B, & \text { if } X_{n} \geq Z_{c} \\
\end{array}$ \\
\hline \multicolumn{3}{|c|}{$G_{n}=\frac{1}{Y_{n}-X_{n}}\left[B\left(Y_{n}-Z_{c}\right)+0.5 Z_{c}^{2}-0.5 X_{n}^{2}+e D \Delta_{c}\left(Z_{c}-X_{n}\right)\right], H_{n}=\frac{0.5\left(Y_{n}-Z_{d}\right)^{2}}{Y_{n}-X_{n}}, Z_{d}=D \Delta_{d}, Z_{c}=B-e D \Delta_{c}$} \\
\hline
\end{tabular}

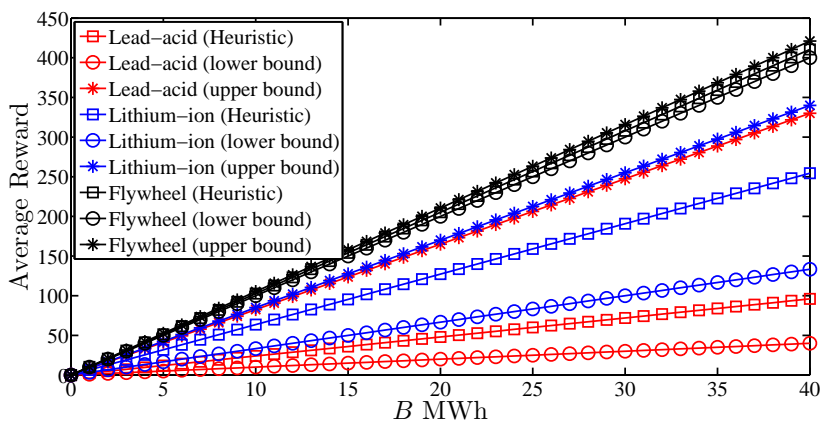

Fig. 2. The expected value of the reward $E\left\{F_{T}\right\}$ (in $\$$ ) as well as the lower and upper bounds on $F_{T}$, as a function of $B$ for $D=1 h$ when $\alpha=10 h$ (Lead-acid), $\alpha=3 h$ (Lithium-ion), $\alpha=2 \min$ (Flywheel), $T=10 h$, $K=60$, and $a_{n}=b_{n}=1 \$ /(\mathrm{h} \mathrm{MW})$.

approximation of the expected reward $E\left\{F_{T}\right\}$ as well as the lower and upper bounds on $F_{T}$, as a function of $B$ for $D=1 h$. Our numerical results in Fig. 2 show that

- The average reward $E\left\{F_{T}\right\}$ is quasi-linear in $B$ for the three technologies.

- The spread between the minimum and maximum reward is small for Flywheel, but greater for the other two technologies.

- Flywheel technology achieves a higher reward for the same value of $B$ than Lithium-ion can, which, in turn, does better than Lead-acid.

We have obtained the same results for different values of $D$.

\section{CONCLUSION}

This paper provides tools to quantify offline the reward a regulation unit with non-ideal storage could expect over multiple contracts. We show the impact of $D$ the contract duration on the expected reward and we compare several storage technologies in terms of the reward they can expect.

\section{APPENDIX: PROOF OF PROPOSITION 2}

Given $D, e$, and $B$, let us define the linear functions $P\left(U_{n}\right)=\frac{U_{n}}{D}$ and $Q\left(U_{n}\right)=\frac{B-U_{n}}{D e}$ where $U_{n} \in[0, B]$. The SRU reward for contract $n$ depends on the values of the parameters $\Delta_{c}, \Delta_{d}, D, e$, and $B$, and on the value of $U_{n}$ in contract $n$. We consider the following cases:

Case 0: Since $\Delta_{d} \geq \Delta_{c}$ and $e \leq 1$, it is impossible to have $\Delta_{d}<\frac{B}{D}$ and $e \Delta_{c} \geq \frac{B}{D}$.

Case 1: Assume that $e \Delta_{c} \geq \frac{B}{D}$ and $\Delta_{d} \geq \frac{B}{D}$. We can verify that the optimal value of $f_{n}\left(R_{n}, r_{n}\right)$ is determined by $\left(a_{n} P\left(U_{n}\right)+b_{n} Q\left(U_{n}\right)\right) \times D$ irrespective of the value of $U_{n}$, and hence the lower and upper bounds of the reward are equal to $\left(B \min \left\{a_{n}, \frac{b_{n}}{e}\right\}\right)$ and $\left(B \max \left\{a_{n}, \frac{b_{n}}{e}\right\}\right)$, respectively.

Case 2: Assume that $\Delta_{d} \geq \frac{B}{D}$ and $e \Delta_{c}<\frac{B}{D}$. Let us define $Z_{c}=\left(B-\Delta_{c} e D\right)$. Note that we have $0 \leq Z_{c} \leq B$. Given $\Delta_{c}$, $\Delta_{d}, D, e$, and $B$, depending on the value of $U_{n}$ in contract $n$, the SRU reward for contract $n$ is determined by one of the following cases:

- If $Z_{c} \leq U_{n} \leq B$, then $P\left(U_{n}\right) \leq \Delta_{d}, Q\left(U_{n}\right) \leq \Delta_{c}$, and $f_{n}\left(R_{n}, r_{n}\right)=\left(a_{n} P\left(U_{n}\right)+b_{n} Q\left(U_{n}\right)\right) \times D$.

- If $0 \leq U_{n} \leq Z_{c}$, then $P\left(U_{n}\right) \leq \Delta_{d}, Q\left(U_{n}\right) \geq \Delta_{c}$, and $f_{n}\left(R_{n}, r_{n}\right)=\left(a_{n} P\left(U_{n}\right)+b_{n} \Delta_{c}\right) \times D$.

Let $W_{n}=\left(b_{n}+a_{n}\left(\frac{\alpha \eta}{D}-e\right)\right) D \Delta_{c}$. The reward in contract $n$ is bounded as follows:

- If $Z_{c} \leq U_{n} \leq B$, then $\min \left\{a_{n} B, W_{n}\right\} \leq f_{n}\left(R_{n}, r_{n}\right) \leq$ $\max \left\{a_{n} B, W_{n}\right\}$.

- If $0 \leq U_{n} \leq Z_{c}$, then $b_{n} \Delta_{c} D \leq f_{n}\left(R_{n}, r_{n}\right) \leq W_{n}$.

Note that, in this case, we have $W_{n} \geq b_{n} \Delta_{c} D$.

Proofs for the following cases are omitted due to space limitations:

- Case 3: $\Delta_{d}<\frac{B}{D}, e \Delta_{c}<\frac{B}{D}$, and $Z_{c} \leq Z_{d}$

- Case 4: $\Delta_{d}<\frac{B}{D}, e \Delta_{c}<\frac{B}{D}$, and $Z_{c}>Z_{d}$.

\section{REFERENCES}

[1] Y. V. Makarov et al., "Assessing the Value of Regulation Resources Based on Their Time Response Characteristics", Pacific Northwest National Laboratory, PNNL-17632, June 2008.

[2] Y.V. Makarov et al., "Wide Area Energy Storage and Management System to Balance Intermittent Resources in the Bonneville Power Administration and California ISO Control Areas," PNNL-17574, Pacific Northwest National Laboratory, Richland, WA, 2008.

[3] Available at www.ercot.com/content/gridinfo/etts/keydocs/ERCOT_ Storage_Issues_Whitepaper.doc.

[4] V. Pandurangan et al., "Frequency regulation services: A comparative study of select North American and European reserve markets," North American Power Symposium (NAPS), pp. 1-8, Sept. 2012.

[5] M. Swierczynski et al., "Primary Frequency Regulation with Li-ion Battery Energy Storage System: a Case Study for Denmark," ECCE Asia, pp. 487-492, June 2013.

[6] J. T. Alt et al., "Assessment of utility side cost savings from battery energy storage," IEEE Trans. Power Syst., vol. 13, no. 3, pp. 1112-1120, Aug. 1997.

[7] A.D. Papalexopoulos, and P.E. Andrianesis, "Performance-Based Pricing of Frequency Regulation in Electricity Markets," IEEE Trans. on Power Systems, Dec. 2012.

[8] N. Lu et al., "Evaluation of the flywheel potential for providing regulation service in California," Power and Energy Society General Meeting, 2010.

[9] A. Oudalov et al., "Optimizing a Battery Energy Storage System for Primary Frequency Control," IEEE Trans. on Power Systems, vol. 22, no. 3, pp. 1259-1266, Aug. 2007.

[10] Available at http://batteryuniversity.com.

[11] D. Wang et al., "Energy storage in datacenters: what, where, and how much?," in Procc. of ACM SIGMETRICS/PERFORMANCE, pages 187198, June 2012. 